\title{
A Research Study Review of Effectiveness of Treatments for Psychiatric Conditions Common to End-Stage Cancer Patients: Needs Assessment for Future Research and an Impassioned Plea - A Commentary and Controversy
}

\author{
Ralph J Johnson* \\ Department of Lymphoma/Myeloma, Texas Medical Center Catholic Chaplaincy Corp, Houston, USA
}

\begin{abstract}
The end stage cancer involves particular psychiatric conditions and their treatments that have varying degrees of effectiveness. This Commentary article covers a recent comprehensive and detailed literature review research study report centered on treatment effectiveness that also includes a methodological controversy and its resolution in which the study was involved. The resolution of this methodological controversy and mixed-method approach has far reaching implications for how these kind of studies are conducted in the future. Which have substantial power to inform mental health administration and policy making. This commentary also reviews important highlights from the studies' conclusions.
\end{abstract}

Keywords: Psychiatric services; Psycho-oncology; End stage cancer care; Needs assessment methods; PRISMA; Qualitative methods

\section{INTRODUCTION}

Johnson reports on a comprehensive 5-phase iterative needs assessment study [1]. The study was systematic, evidence-based and thematically grounded. This study's results suggest administrative and policy recommendations for the most effective use of treatments for psychiatric conditions common to end-stage cancer patients (Figure 1 for pictorial definition of where end-stage cancer care lies on the continuum of cancer care) [1-5].

End-stage cancer care constitutes the terminal phase of cancer including hospice and the latter part of palliative care [3-13]. What was of most importance about this study was the methodological yet untold story through which conclusions about mental health administration and policy-making were derived. This as-yet untold story has far-reaching implications that extend into future program evaluation and possibly the very process of scientific literature review as research inquiry.

(1) To contend that rates of particular psychiatric conditions (delirium, depression, anxiety disorders) have been naturally high and common to end-stage cancer, and remain unchanged [1420]. Yet the number of patients suffering from them is increasing dramatically, as populations are aging and chronic cancer is a chief cause of debility and mortality in aging populations [16,17,19-22]. Therefore, a review of what has been substantiated in the research literature regarding efficacy-that is, best practices for treatment

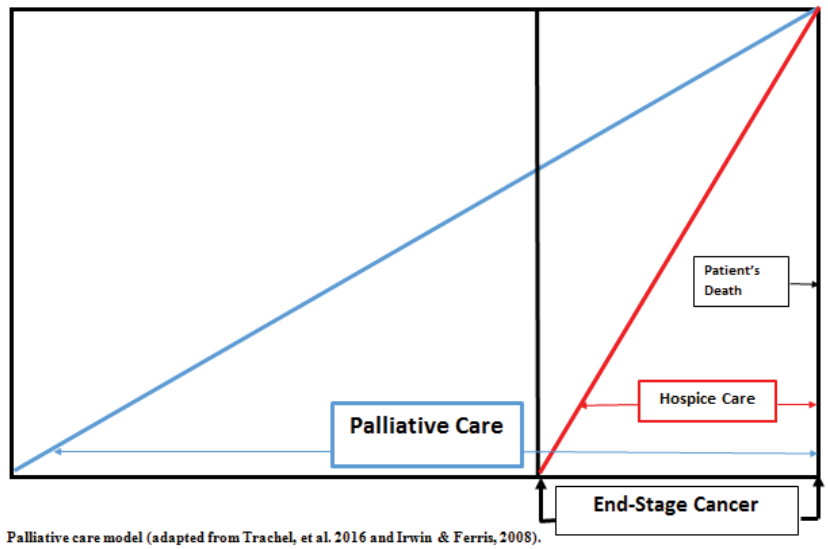

Figure 1: End stage cancer on the terminal cancer care continuum.

interventions was imperative to evaluate, inform and improve these patients' mental healthcare $[7,19,21,23,24]$. This should be an impending concern to all of us, as the probability is high we will eventually suffer such conditions.

(2) To report results from a current comprehensive evidence-based investigation and analyses that intended to assess the state of research regarding treatment effectiveness and the need for future research [1]. This review study drew on previous seminal studies as well as a few on-going studies regarding psychiatric treatment effectiveness for end-stage cancer patients. Moreover, it reported

${ }^{*}$ Correspondence to: Ralph J Johnson, Department of Lymphoma/Myeloma, Texas Medical Center Catholic Chaplaincy Corp, Houston, USA, Tel: 832 372-3511; E-mail: rjjohnson@mdanderson.org; jayjohnson131313@gmail.com

Received: January 17, 2019; Accepted: January 27, 2019; Published: February 02, 2019

Citation: Johnson RJ (2019) A Research Study Review of Effectiveness of Treatments for Psychiatric Conditions Common to End-Stage Cancer Patients: Needs Assessment for Future Research and an Impassioned Plea - A Commentary and Controversy. Health Care Current Reviews 7: 237. doi: $10.35248 / 2375-4273.19 .07 .237$

Copyright: (C) 2019 Johnson RJ. This is an open-access article distributed under the terms of the Creative Commons Attribution License, which permits unrestricted use, distribution, and reproduction in any medium, provided the original author and source are credited. 
on fairly recent works that expanded and extended them [24-31]. Furthermore, unlike previous studies, this one included emerging scientific studies of the recent growing field of non-traditional/ alternative mental health therapies and spirituality interventions [32-34].

(3) To provide integrated descriptive results from a comprehensive, representative, systematic and iteratively grounded method and analysis for identifying studies and data, and to enumerate themes for inclusion $[35,36]$. This process was employed because it lent itself to the sine wed and synthesized crafting of the end-product $[35,36]$. More importantly, grounded method and theory selfacknowledges a healthy modicum of equipoise in terms of its own limitations, when accounting for findings produced [35-37].

First, since psychiatric symptoms and disorders among end-stage cancer patients have remained unchanged over time, there can be a tendency for adherents of conventional paradigms to argue that treatments, policies, and mental health care administration likewise should remain relatively static $[2,6,14,18,25,31,38]$. Yet, patients 65 years and older are the fastest-growing populations, and have the highest rates of end-stage cancer [10,31,39]. Not only do surrounding psychiatric conditions cause inordinate suffering in their own right, but they also cause physical debility and degrade quality of life-a problem that end-stage cancer care aims to ameliorate [12,40-43]. Therefore, the treatment of end-stage cancer patients' psychiatric conditions is fast approaching a watershed moment in terms of administrative policy implications-all of which must be reformed based on timely and valid information.

Past literature reviews had focused on one or two psychiatric conditions and some of their treatments effectiveness for end-stage cancer patients $[4,6,15,17,19,20,29,44,45]$. Some were more or less up-to-date, and one was on-going and solely devoted to end-stage cancer depression [29]. But few, if any, studies (until this one) have provided a reasonably current, comprehensive detailed review of effectiveness of psychiatric treatments for the range of end-stage cancer patient psychiatric treatments with an eye toward the state of research and need for future research. Hence, the article described herein also reports scientific examinations of newer and even non-traditional/alternative treatments [1]. The ultimate goal was to provide a compendium of "pearls" of best practices for endstage cancer patients' mental health care, so as to help oncologists, mental health practitioners and researchers, and policymaker's better address emotional and psychiatric suffering and improve quality of life for these patients.

Second, in collecting research articles and answering the challenge of integrating descriptive data, this article reports on the use of an evidence-based, 5-phase systematic strategy [1]. The focus of this strategy was a fortuitous grounded qualitative methodology employed iteratively to generate and assign themes (i.e., code), until "thematic saturation" was reached $[35,36]$. Specifically, there was an exhaustion of themes. An inherent risk and explicitly acknowledged limitation of this approach is that there may always be some studies/themes missed. However, the process will produce a general and credible mosaic assessment [35,36]. Also, this process readily lends itself to the construction of an integrated and synthesized end-product consisting of brief yet thick descriptive accounts of, in the case of the study and article described herein: Effectiveness of psychiatric interventions with end-stage cancer patients that also lent itself readily to a needs assessment and stateof-the-research inquiry [37]. This ultimate aim is emblematic of the scientific enterprise. In other words, it is a systematic empirical investigation into what is known, with an eye also on what is not known, and what needs to be known $[46,47]$.

Figure 2 depicts the evidence-based and best practices qualitative process that informed the research study through which opensource articles were identified, reviewed, and conceptual themes generated. Figure 2 illustrates the process by which conceptual themes were identified for inclusion, how/where they would be incorporated, and assessments rendered regarding their relevance, which further drove and reinvigorated the research process (Figure 2, Phase 1 to Phase 2 then back again to Phase 1).

Essentially, the figure conveys the steps in the research process.

Step 1 involved deriving key words to search articles.

Step 2 was removing duplicate articles.

Step 3 was reviewing articles, particularly abstracts, for relevance, and removing irrelevant articles.

Step 4 was assigning research articles themes and inclusion of studies in the evidence-based review. At this point, the process proceeded to the next step, specifically, evaluation, aggregation, and integration according to themes.

Step 5 was an assessment of the psychiatric treatments efficacy in end-stage cancer patients' mental conditions.

Step 6 was ascertaining in terms of treatment effectiveness whether the treatment modality should be sustained, improved/modified, or discarded, and any mental health administration policy implications.

Step 6 was a cycling iteration eventually back to Step 4 .

There is an optional (dashed line) Step 6.5 should new research terms be uncovered, which is in accordance with the Grounded Theory methodology $[35,36]$. Had the work been done initially

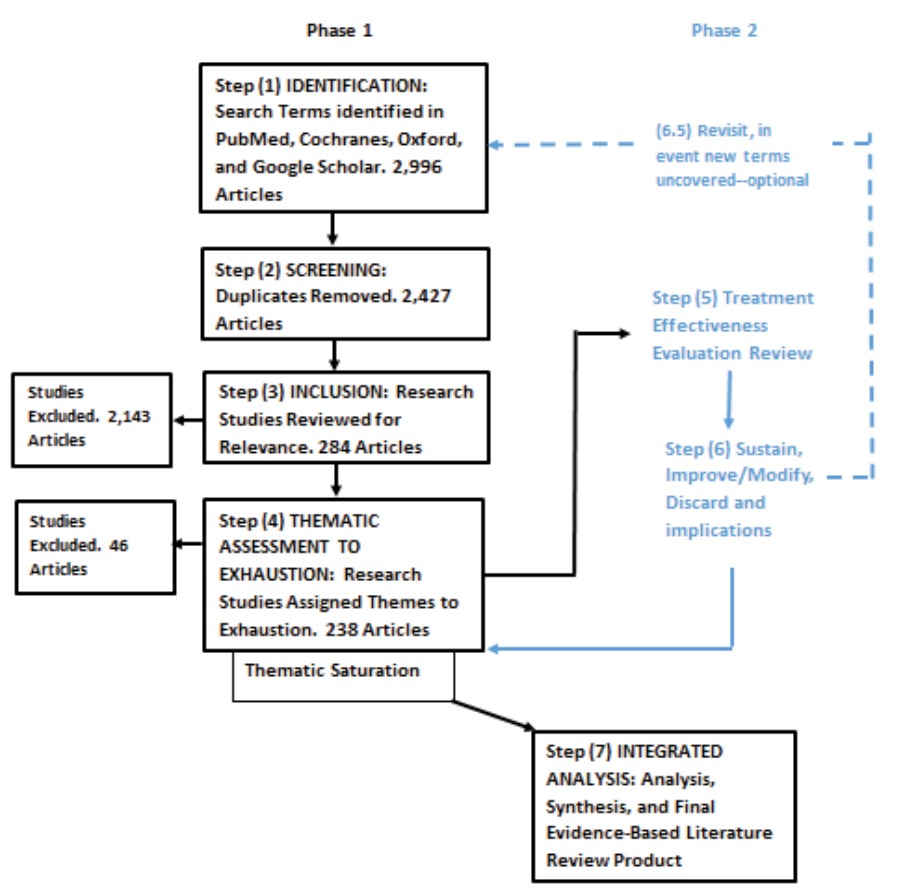

Figure 2: Qualitative Process Informing PRISMA. 
according to PRISMA (i.e., Preferred Reporting Items of Systematic Review and Meta-analysis), this step will probably not be necessary $[48,49]$.

Step 7, with the realization that thematic saturation was reached where all relevant themes had been identified, enumerated, inventoried and synthesized there was a reasonable certainty of a final end-product: A comprehensive descriptive report. This process was cyclic, can be perpetual, and is almost self-explanatory. Through technology transfer, it is applicable to other fields of study of mental health inquiry or programmatic evaluation for needs assessment and administrative policy making.

Note: Steps 1 - 4 generally followed the PRISMA flow chart/ checklist [48-50].

Third, the endeavours in this article involved a ubiquitous and quintessential methodological controversy encompassing not just mental health research, administration and policy but possibly the inherent nature of the science of systematic literature reviews. Specifically, one sector of the study was critical about the limitations of the qualitative method and alternatively pushed to strictly follow the PRISMA protocol favouring numeric collections and/or disembodied inventory lists of study briefs over qualitative assessment, integration, synthesis, and "sense making" [47-50]. Again, the method employed in the study attempts to achieve a healthy equipoise by assuming that it will never achieve ideal thematic saturation. That is, its endpoint is only a general approximation of "what is going on out there." Also, another criticism was that the method used heavily emphasizes researcher involvement in the assessment, interpretation, consolidation, integration, incorporation, synthesis of the "raw" data and report, though this is a fundamental aspect of scholarly, scientific inquiry $[37,47,48]$. Of course, every additional step between data and product involves the risk of introducing bias, which the method acknowledges [36].

The advent of PRISMA resulted from the recent technological development of electronic, high-tech, sophisticated computerized digital search engines and sizable scientific literature databases: Google Scholar, Pub Med, Cochranes, Medscape, Oxford, etc $[48,50]$. In response, the PRISMA guidelines initiative recognized the increasing importance of large-scale literature reviews and macro/meta analyses for influencing healthcare practice and policy making and for practitioners to remain current in their fields $[48,50]$. PRISMA standards consist of checklists and flow charts deemed by PRISMA developers as essential for the full reporting of a truly systematic review [48-51].

During the development of the review study reported herein, in the interest of ensuring and reporting representativeness and comprehensiveness in terms of article inclusion, the PRISMA practical flow-chart was adhered to as a guideline, especially in Steps 1-3. However, rather than enumerating articles and their numbers, the review study's objective was identifying and describing themes in articles generated from the sifting process of including/ excluding articles. Also, merely enumerating articles is not only irrelevant but deceptive in that some articles represent more than one type of psychiatric condition and may involve multiple types of treatments, some in combination $[5,7,9,11,12,14,16-18,31,52]$. Simply counting articles as singular units of measure might either dilute or risk double-counting with a bias toward under- or overinflation of importance.
As the PRISMA protocol for accurate article generation was generally followed the depiction conveyed a rough estimate of representativeness and comprehensiveness of the review of the literature as well as the Herculean efforts involved in the purity of scholarship. Yet, the use of the PRISMA protocol flow sheet as only a model and not the core of the analysis was a compromise. The PRISMA approach appears to favour sheer counting and not qualitative assessment; it even provides a disclaimer that "(It) is not intended to be a qualitative assessment, and therefore it should not be used as such" so for this study it was not used analytically [51].

Both Grounded Methodology and PRISMA aim at a representativeness of the state of scientific inquiry in the scientific literature but they are worlds apart in how to do it. PRISMA to its credit is a general standard for systematic and comprehensive collection of study documents. Nevertheless, until PRISMA methodologically incorporates qualitative assessments in its guidelines, blind and uniformed use appears to lend itself to:

(1) Disembodied inventories (i.e., laundry lists) of study briefs that search engines already are capable of churning out; and/or

(2) Statistical analysis on studies as units of data without regard to the meaning of those units of data. In contrast, qualitative Grounded Theory and methods maintain a healthy, prudent respect and nagging self-doubt when enumerating and describing every nuance and pattern, without necessarily assigning numeric weight and importance to any.

Though well-intentioned in terms of addressing sub-optimal reporting of meta-analysis, PRISMA could lead to erroneous results that are similar to numeric research involving abstracted archival records, as both have similar characteristics $[48,53,54]$. The risks of using archived documents including research articles numerically/ statistically were described several years ago and should be kept in mind when conducting numeric/statistical analysis on them as units of data-no matter the methodology. There are several reasons for this.

One, they were probably produced for aims and agendas other than originally intended, and that bias can be inflated through numeric/statistical analysis. Two, there may be patterns of missing items leading to bias. Three, there may be inaccuracies due to the mediums through which they are conveyed-some of which are irreconcilable. Four, they may represent other needs, e.g., bureaucratic or financial, besides research. For example, the number of articles may arbitrarily represent funding streams rather than importance or changes in dependent variables. Fifth, no matter whether Grounded Methodology, PRISMA or whatever protocol is employed, literature reviews and researching articles en masse is by far the least intrusive and least costly way to conduct high-quality scholarly scientific inquiry.

\section{DISCUSSION}

In sum, the most profound implication of the research work reported herein is that combined processes should be incorporated into future reviews of treatment of end-stage cancer patients' psychiatric treatments. To its credit, combining grounded methodology with PRISMA as such resulted in an economy of action and the PRISMA process was similar to Grounded Theory's purposive, non-probabilistic theoretic sampling to ensure rich information 
to contribute to the study [36]. The two should be employed practically to inform other health care disciplines beyond mental healthcare and oncology in terms of best practices for treatments and policy initiatives. In terms of treatments for end-stage cancer patients' psychiatric conditions, the identification and existence of voids in information demands remediation and restitution through the delivery of high-quality, peer-reviewed, evidence based and best-practices findings. With these, planning and policy making administrators can craft relevant and high-impact programmatic modifications and initiatives.

Despite the controversies encountered in the study, there were several relevant and high-impact implications that mental health practitioners and administrators should take under advisement. Foremost, the study revealed a testable linear model of a combination of psychiatric treatments for communicative and cognizant end-stage cancer patients, depicted in Figure 3.

This model has correlative models that emerged through rigorous scientific review of the cost-effectiveness of treatments on other psychiatric patient populations (Note: The weight or importance of co-factors is displayed by their order of precedence, left to right) [53]. First, in accordance with best-practice guidelines, the goal of psychiatric treatments for end-stage cancer patients is reductions in psychiatric symptoms and enhancement of their quality of life [12,54-58]. Second, depending on symptoms, medications are the most powerful tools in the psychiatric arsenal, and this armory is expanding and extending with new editions and offlabel combinations [5]. Third, some form of psychological ("talk") counselling/intervention appears to support and augment psychiatric medication interventions [59-62]. Though their effectiveness might be mixed, common sense dictates that the potential harm is relatively minimal and they can serve as vehicles for fortifying the therapeutic alliance. Fourth, though a connection between psychosocial education and psychiatric therapeutic effectiveness has yet to be established, such education has some beneficial effects for patients in crises such as end-stage cancer; thus research on it should not be abandoned entirely $[14,15,29,63,64]$. Fifth studies on interventions designed to enhance spirituality have shown substantially promising results in terms of quality of life and should be considered for inclusion in the constellation of future psychiatric treatment planning $[3,15,16,65-67]$.

Specifically, the complicated and tricky part of each component in the model is negotiating, balancing, conforming, and/or fitting the right treatment with the right patient and diagnosis dose for response, titration and tapering, drug-drug interactions, route of administration, and context in a decisive and timely and cost-effective manner $[4,6,16,31,68]$. Varying amounts of
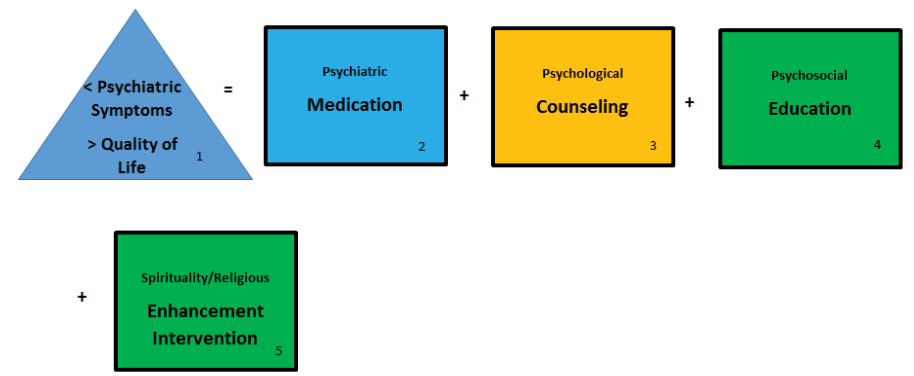

Figure 3: Linear Model for Informing/Combining End-Stage Cancer Patients' Treatments. quality scientific evidence exists regarding some factors that constitute the components in the model. The complicated part of all this is practitioners translating the study's findings into effective and empowering patient care. Fortunately, there is a preeminent parsimonious prototype found in cardio-vascular and endocrine medicine that end-stage cancer patient psychiatric care management can emulate, namely the Leonardo Project [69]. Some of its best-practices features can be found in various disparate psychiatric care approaches in terms of end-stage cancer patients [3-5,7,11,12,14-19,29,30,32-34,44,47,52]. Nevertheless, the Leonardo Project in terms of cardio-vascular and diabetes disease treatment put them altogether in an extremely streamlined, scientifically informed, systematic and logical, and cost-effective strategic approach [69]. Using dedicated computer-ware to collect real-time data, the Leonardo Project conformed medical care management to guidelines in accordance with patients' conditions improving care, while promoting appropriate resource utilization $[4,5,11,12,14,17,19,23,30,69]$. Its vetting proved both viable and highly effective as it informed care managers in bridging the link between patients' conditions and their illness, while tracking effectiveness and resource utilization [7,11,15,16,19,23,30,32,34,69]. The Leonardo system advanced the paradigm of enhancing patients' health knowledge, self-management, care compliance, and thus, empowering patients into their role as the most important and central members of the healthcare team in collaboration with medical practitioners $[3,7,11,12,15,16,19,23,69]$.

Nevertheless, the review study reported herein noted that for some components of the linear model there is an absence of evidence or worse, pseudo-evidence and that much more rigorous and largerscale scientific studies must be conducted. This is particularly true for research on end-stage cancer patients in general and newly developed treatments and off-label combinations in particular and of course, effectiveness studies that include psychiatric intervention costs $[5,53,69]$.

Finally as cancer treatments become more aggressive and successful, and as the populations of older adults grow-and by extension the populations with end-stage cancer-the quality of life for end-stage cancer patients is of paramount importance. Interestingly, a small proportion of patients in this category (approximately 3\%) already suffered from severe and persistent psychiatric conditions, and their end-stage cancer was adjunct to that. This group is expected to grow as the population ages as well, and its psychiatric treatment must be addressed. The general consensus has been that quality research with which to make informed practice and policy decisions is wanting [7,70-75].

\section{REFERENCES}

1. Johnson RJ. A research study review of effectiveness of treatments for psychiatric conditions common to end-stage cancer patients: Needs assessment for future research and an impassioned plea. BMC Psychiatry. 2018;18(85):1-16.

2. Irwin SA, Rao S, Bower KA, Palica J, Rao SS, Maglione JE, et al. Psychiatric issues in palliative care recognition of depression in patients enrolled in hospice care. J Palliat Med. 2008;11(2):158-163.

3. Dein S. Psychiatric liaison in palliative care. Adv Psychiatr Treat. 2003;9(4):241-248.

4. Rodin G, Katz M, Lloyd N, Green E, Mackay JA, Wong RKS, et al. Treatment of depression in cancer patients. Curr Oncol. 2007;14(5):180-188. 
5. Masman AD, van DM, Tibboel D, Baar FP, Mathot RA. Medication use during end-of-life care in a palliative care centre. Int J ClinPharmacol. 2015;37(5):767-775.

6. Marks S, Heinreich T. Assessing and treating depression in palliative care patients. Curr Psychiatry. 2013;12(8):37-40.

7. Ellison N. Mental Health and Palliative Care: A Literature Review. 2008.

8. Kim SH, Shin DW, Kim SY, Yang HK, Nam E, Park JH, et al. Terminal versus advanced cancer: Do the general population and health care professionals share a common language? Cancer Res Treat. 2016;48(2):759-767.

9. Garcia C, Lynn R, Breitbart W. Psychotropic medications in Palliative Care. Primary Psychiatry. 2009;16:25-32.

10. Yanick R, Reis LA. Cancer in older persons: An international issue in an aging world. Seminal Oncolology. 2004;31:125-296.

11. Farriols C, Ferrandez O, Planas J, Ortiz P, Mojal S, Ruiz AI. Changes in the prescription of psychotropic drugs in the palliative care of advanced cancer patients over a 7 year period. J Pain Symptom Manage. 2012;43(5):945-952.

12. Fairman N, Irwin SA. Palliative care psychiatry: Update on an emerging dimension of psychiatric practice. Curr Psychiatry Rep. 2013;15(7):374.

13. Grabsch B, Clarke DM, Love A, McKenzie DP, Snyder RD, Bloch $\mathrm{S}$, et al. Psychological morbidity and quality of life in women with advanced breast cancer: A cross-sectional survey. Palliat Support Care. 2006;4(1):47-56.

14. Miovic M, Block S. Psychiatric disorders in advanced cancer. Cancer. 2007;110(8):1665-1673.

15. Dauchy S, Dolbeault S, Reich M. Depression in cancer patients. EJC Supplements. 2013;11(2):205-215.

16. Irwin SA, Ferris FD. The opportunity for psychiatry in palliative care. Canadian J Psychiatry. 2008;53(11):53.

17. Wilson KG, Chochinov HM, Skirko MG, Allard P, Chary S, Pierre RG, et al. Depression and anxiety disorders in palliative cancer care. J Pain Symptom Manage. 2007;33(2):118-129.

18. Thekdi SM, Trinidad A, Roth A. Psychopharmacology in cancer. Curr Psychiatry Rep. 2015;17(529):1-10.

19. Jacobsen PB, Donovan KA, Swaine ZN, Watson IS. Management of Anxiety and Depression in Adult Cancer Patients: Toward an Evidenced-Based Approach. Oncology. 2006;1561-1588.

20. Ginsburg A. Cancer-related depression and potential pharmacologic therapies. Proc Bay Univ Med Cent. 2008;21(4):439-441.

21. Breitbart W, Freidlander M, Lawlor P. Delirium issues in the terminally. 2010.

22. Fisch M. Treatment of depression in cancer. J Nat Cancer Inst Monographs. 2004;2004(32):105-111.

23. Krebber AM, Jansen F, Witte BI, Cuijpers P, de Bree R, BeckerCommissaris A, et al. Stepped care targeting psychological distress in head and neck cancer and lung cancer patients: A randomized, controlled trial. Ann Oncology. 2016;27(9):1754-1760.

24. Attard A, Ranjith G, Taylor D. Alternative route to oral antidepressant therapy: Case vignettes and literature review. Psychopharmacology. 2010;24(4):449-454.

25. Hotopf M, Chidgey J, Addington HJ, Ly KI. Depression in advanced disease: A systematic review Part 1 Prevalence and case finding. Palliat Med. 2002;16:81-97.

26. LeGrand SB. Delirium in palliative medicine: A review. J Pain
Symptom Manage. 2012;44(4):583-594.

27. Callari A, Mauri M, Miniati M, Mancino M, Bracci G. Treatment of depression in patients with breast cancer: A critical review. Tumori. 2013;99:623-633.

28. Muriel AC, Hwang VS, Kornblith A, Greer J, Greenberg DB. Jennifer $\mathrm{T}$, et al. Management of psychosocial distress by oncologists. Psychiatr Serv. 2009;60(8):1132-1134.

29. Breitbart W, Dickerman AL. Assessment and Management of Depression in Palliative Care. 2017.

30. Trachsel M, Irwin SA, Biller AN, Hoff P, Riese F. Palliative psychiatry for severe persistent mental illness as a new approach to psychiatry? Definition, scope, benefits, and risks. BMC Psychiatry. 2016;16:1-6.

31. Winell J, Roth AJ. Psychiatric assessment and symptom management in elderly cancer patients. Oncology. 2005;19(11):1479-1490.

32. Kienle GS, Glockmann A, Schink M, Kiene H. Viscum album L. extracts in breast and gynaecological cancers: A systematic review of clinical and preclinical research. J Exp Clin Cancer Res. 2009;11:79.

33. Pilkington K, Kirkwood G, Rampes H, Fisher P, Richardson J. Homeopathy for anxiety and anxiety disorders: A systematic review of the research. Homeopathy. 2006;95(3):151-162.

34. Su CX, Wang LQ, Grant SJ, Liu JP. Chinese herbal medicine for cancer-related fatigue: A systematic review of randomized clinical trials. Complement Ther Med. 2014;22(3):567-579.

35. http://www.sxf.uevora.pt/wp-content/uploads/2013/03/ Glaser_1967.pdf

36. Corbin J, Strauss A. Basics of Qualitative Research: Techniques and Procedures for Developing Grounded Theory. 2015.

37. https://us.sagepub.com/en-us/nam/constructing-grounded-theory/ book 235960

38. Fitzgerald P, Miller K, Li M, Rodin G. Depressive Disorders. PsychoOncology. 2015.

39. Rao A, Cohen HJ. Symptom management in the elderly cancer patient: Fatigue, pain, and depression. J Nat Cancer Inst. 2004;32:150-157.

40. Greer JA, Jackson VA, Meier DE, Temel JS. Early integration of palliative care services with standard oncology care for patients with advanced cancer. Cancer J Clin. 2013;63(5):349-363.

41. Ferrell BR, Temel JS, Temin S, Alesi ER, Balboni TA, Thomas JS, et al. Integration of palliative care into standard oncology care: American Cancer Society of clinical oncology clinical practice guideline update. J Clin Oncology. 2017;35(1):96-112.

42. Ferris FD, Balfour HM, Bowen K, Farley J, Hardwick M, Claude L, et al. A model to guide patient and family care: Based on nationally accepted principles and norms of practice. J Pain Symptom Manage. 2002;24(2):106-123.

43. Inglewicz A, Morrison K, Nelesen RA, Tingting Z, Iglewicz B, NathanFairman, et al. Ketamine for the treatment of depression in patients receiving hospice care: A retrospective chart review of thirtyone cases. Psychosomatics. 2015;56(4):329-337.

44. Morita T. Palliative sedation to relieve psycho-existential suffering of terminally ill cancer patients. J Pain Symptom Manage. 2004;28(5):445450.

45. https://ecommons.cornell.edu/handle/1813/83

46. http://strangebeautiful.com/other-texts/popper-logic-scientificdiscovery.pdf

47. http://prisma-statement.org/PRISMAStatement/ HistoryAndDevelopment

48. Tricco AC, Lillie E, Zarin W, O’Brien KK, Colquhoun H, Levac D, et 
al. PRISMA Extension for Scoping Reviews (PRISMA-ScR): Checklist and Explanation. Ann Int Med Res Rep. 2018:169(7):467-473.

49. Liberati A, Altman DG, Tetzlaff J, Mulrow C, Gotzsche PC, John PAI, et al. The PRISMA statement for reporting systematic reviews and meta-analysis of studies that evaluate health care interventions: Explanation and elaboration. Plos Med. 2009;6:1-27.

50. Moher D, Liberati A, Tetzalff J, Altman DG. PRISMA Group Preferred reporting items for systematic reviews and meta-analyses: The PRISMA statement. J Clin Epidemiol. 2009;62:1006-1012.

51. Cankurtaran ES, Ozalp E, Soygur H, Akbiyik DI, Turhan L. Mirtazapine improves sleep and lowers anxiety and depression in cancer patients: Superiority over imipramine. Supp Care Cancer. 2008;16(7):1291-1298.

52. Dworkin RJ. Hidden Bias in the Use of Archival Data. Evalu Health Prof. 1987;10:173-185.

53. Dworkin RJ. Alternative data sources in Researching Persons with Mental Illness. App Soc Res Meth series. 1999.

54. Johnson RJ. Cost-effectiveness of mental health services for persons with a dual diagnosis: A literature review and the CCMHCP. J Subst Abuse Treat. 2000;18(2):119-127.

55. Kvale E, Azuero CB, Walker E. Depression and Fatigue. 2013.

56. http://www.cancer.org/treatment/findingandpayingfortreatment/ understandingfinancialandlegalmatters/advancedirectives/advancedirectives-why-do-we-need-advancedirectives

57. Tan WW (ND). Palliative Cancer Care Guidelines.

58. Kissane DW. The contribution of demoralization to end of life decision making. Hastings Cent Rep. 2004;34(4):21-31.

59. Schuman OZ, Bendel DH, Forstein M, Price BH. The use of palliative sedation for existential distress: A psychiatric perspective. Harv Rev Psychiatry. 2008;16(6):339-351.

60. Breitbart W, Rosenfeld B, Gibson C, Pessin H, Poppito S, Nelson $\mathrm{C}$, et al. Meaning centered group psychotherapy for patients with advanced cancer: A pilot randomized controlled trial. Psychooncology. 2010;19(1):21-28.

61. Breitbart W, Poppito S, Rosenfeld B, Vickers AJ, Li Y, Abbey J, et al. Pilot randomized controlled trial of individual meaning-centered psychotherapy for patients with advanced cancer. J Clin Oncol. 2012;30(12):1304-1309.

62. Spiegel D, Bloom JR. Group therapy and hypnosis to reduce breast cancer carcinoma pain. Psyhcosom Med. 1983;45:333-339.

63. Spiegel D, Bloom JR, Yalom I. Group support for patients with metastic cancer: A randomized outcome study. Arch of Gen Psychiatry. 1981;38:527-533.

64. Osborn RI, Demoncada AC, Feurestein M. Psychosocial interventions for depression and quality of life in cancer survivors: Meta-analysis. Int J Psychiatry Med. 2006;36(1):13-34.

65. Wilson KG, Chochinov HM, de Faye BJ, Breitbart W. Diagnosis management in depression in pailliative care. 2000.

66. Breitbart W, Gibson C, Poppito S, Berg A. (2004) Psychotherapeutic Interventions at the end of life: A focus on meaning and spirituality. Canadian J Psychiatry. 2004;49(6):366-372.

67. Wang CW, Chow AY, Chan CL. The effects of life review interventions on spiritual well-being, psychological distress, and quality of life in patients with terminal or advanced cancer: A systematic review and meta-analysis of randomized controlled trials. Palliat Med. 2017.

68. Dein S. Transcultural psychiatry. British J Psychiatry. 2002;181(6):535536.

69. Barnhill JW, Dickerman AL. DSM-5 and Psycho-oncology. 2015.

70. Ciccone MM, Aquilino A, Cortese F, Scicchitano P, Sassara M, Rollo R, et al. Feasibility and effectiveness of a disease and care management model in the primary health care system for patients with heart failure and diabetes (Project Leonardo). Vasc Health Risk Manag. 2010;6(1):297-305.

71. Davie E. A social work perspective on palliative care for people with mental health problems. Eu J Palliat Care. 2006;13:26-28.

72. McCasland LA. Providing hospice and palliative care to the seriously and persistently mentally Ill. J Hospice Palliat Nurs. 2007;9:305-313.

73. Kelly BD, Shanley D. Terminal illness and schizophrenia. J Palliat Care. 2000;16:55-57.

74. https://books.google.com/books/about/Death_Dying_and_Social_ Differences.html?id=ExUfeLElCR8C

75. Addington HJ. Positive Partnerships: Palliative case for adults with severe mental health problems. Handbook of Palliative Care. 2000. 